GONORRHOEA

\title{
The epidemiology of ciprofloxacin resistant isolates of Neisseria gonorrhoeae in Scotland 2002: a comparison of phenotypic and genotypic analysis
}

\author{
H M Palmer, H Young, I M C Martin, C A Ison, B G Spratt
}

Sex Transm Infect 2005;81:403-407. doi: 10.1136/sti.2004.013565

See end of article for authors' affiliations

.....................

Correspondence to: H M Palmer, Scottish

Neisseria gonorrhoeae

Reference Laboratory,

Department of Medical

Microbiology, Royal

Infirmary of Edinburgh, 51

Little France Crescent,

Edinburgh EH16 5SA', UK;

helen.palmer@luht.scot.

nhs.uk

Accepted for publication

26 January 2005
Objectives: To characterise all isolates with reduced susceptibility or resistance to ciprofloxacin received by the Scottish Neisseria gonorrhoeae Reference Laboratory (SNGRL) in 2002 using N gonorrhoeae multiantigen sequence typing (NG-MAST); to compare NG-MAST with conventional typing and to describe the epidemiology of ciprofloxacin resistant gonorrhoea in Scotland in 2002.

Methods: Isolates were characterised on receipt by auxotyping and serotyping (A/S typing), and antibiotic susceptibility testing, and retrospectively by NG-MAST. Epidemiological data were requested for all isolates in the study.

Results: The 106 isolates were separated into more sequence types (ST) than A/S classes (44 versus 17). All isolates within a sequence type had the same serotype, were homogeneous with respect to ciprofloxacin resistance category, but were sometimes heterogeneous with respect to auxotype or plasmid borne resistance to penicillin. Combined NG-MAST and epidemiological data revealed sustained transmission of several gonococcal strains predominantly within Greater Glasgow and Lothian. Clusters of isolates were associated with transmission within the United Kingdom, whereas isolates with unique STs were associated with foreign travel $(p<0.0001)$.

Conclusions: NG-MAST is more discriminatory than A/S typing. Ciprofloxacin resistant gonococcal isolates in Scotland are heterogeneous, with endemic spread of some strains occurring predominantly in Greater Glasgow and Lothian.
$\mathrm{H}$ igh levels of ciprofloxacin resistant gonorrhoea have been reported worldwide, initially in the South East Asia and Pacific regions in the early 1990s, ${ }^{1}$ but more recently in the United Kingdom, ${ }^{2-3}$ parts of Europe, ${ }^{4-5}$ and some regions of America. ${ }^{6}$ In 2002 the prevalence of ciprofloxacin resistant Neisseria gonorrhoeae in Scotland rose above $5 \%$ for the first time, prompting a change in first line therapy from ciprofloxacin to third generation cephalosporins. $^{3}$ Alongside antibiotic resistance surveillance, which informs timely change in therapy, it is also important to understand the epidemiology of gonorrhoea, particularly of those strains exhibiting antibiotic resistance. Details of the extent of spread of particular gonococcal strains and their association with specific human population groups can be used to direct the use of resources to limit the spread of gonorrhoea, including infections caused by resistant isolates.

The analysis of sexual networks by classic epidemiological approaches (partner tracing) is limited by the information that patients can provide, whereas comparison of the infecting gonococcal isolates permits a much more comprehensive picture. The combination of epidemiological and typing data, is potentially much more useful, being limited only by the completeness of the strain collection that is analysed and level of discrimination offered by the typing method used.

The Scottish Neisseria gonorrhoeae Reference Laboratory receives isolates from nearly all the episodes of gonorrhoea that are diagnosed in Scotland, and epidemiological data (including patient sexual orientation and country of acquisition of the infection) are sought for all isolates that demonstrate antibiotic resistance. Strain characterisation is also routinely performed, which provides an indication of strain heterogeneity and distribution. Until recently characterisation was by means of auxotype and serovar (A/S) classification. However, the discrimination achieved by A/S typing is known to be limited and more discriminatory sequence based methods are now available. ${ }^{7-9}$ Of these currently available sequence based methods $N$ gonorrhoeae multi-antigen sequence typing (NG-MAST) ${ }^{9}$ is most advantageous because it generates a simple numerical sequence type (ST) from the combined sequence data of two genes (por and $t b p B$ ) by means of an internationally accessible web based data analysis system.

The aims of this study were (a) to compare the discrimination of conventional A/S typing with NG-MAST using all $N$ gonorrhoeae isolates from Scotland in 2002, which exhibited reduced susceptibility or resistance to ciprofloxacin, and (b) to describe the epidemiology of these isolates.

\section{METHODS}

\section{Bacterial strains}

Gonoccocal strains submitted to the SNGRL throughout 2002, which exhibited reduced susceptibility (minimum inhibitory concentration $(\mathrm{MIC}) \geqslant 0.125-<1 \mathrm{mg} / \mathrm{l}$ ) or resistance $(\mathrm{MIC} \geqslant 1 \mathrm{mg} / \mathrm{l}$ ) to ciprofloxacin, were used in this study. All isolates were grown on modified New York City medium (Biomerieux, Basingstoke, UK) at $37^{\circ} \mathrm{C}, 5 \% \mathrm{CO}_{2}$ overnight before characterisation.

\section{Phenotypic analysis}

Routine analysis was carried out on receipt of isolates and provided identification as $\mathrm{N}$ gonorrhoeae, antibiotic susceptibility,

\footnotetext{
Abbreviations: $\mathrm{A} / \mathrm{S}$, auxotype/serovar; MIC, minimum inhibitory concentration; NG-MAST, Neisseria gonorrhoeae multi-antigen sequence typing; PPNG, penicillinase producing $N$ gonorrhoeae; SNGRL, Scottish Neisseria gonorrhoeae Reference Laboratory; ST, sequence types; TRNG, tetracycline resistant $N$ gonorrhoeae
} 
serotype and auxotype data. The identification profile included an acidometric test for $\beta$ lactamase production. ${ }^{10}$ Antibiotic sensitivity to six antibiotics (azithromycin 0.015-2 mg/l, ceftriaxone $0.015-0.12 \mathrm{mg} / \mathrm{l}$, ciprofloxacin $0.004-1 \mathrm{mg} / \mathrm{l}$, penicillin $0.015-2 \mathrm{mg} / \mathrm{l}$, spectinomycin $16-128 \mathrm{mg} / \mathrm{l}$, and tetracycline $0.25-4 \mathrm{mg} / \mathrm{l}$ ) was measured using the agar dilution method. ${ }^{11}$ E-tests (AB Biodisk, Sweden) were also carried out for those isolates with ciprofloxacin MIC values $\geqslant 0.125 \mathrm{mg} / \mathrm{l}$, or MIC values for other antibiotics that extended beyond the dilution series. Isolates with a penicillin MIC value $>2 \mathrm{mg} / \mathrm{l}$ and/or a positive acidometric test were tested for $\beta$ lactamase activity using the nitrocephin test. Isolates were serotyped using the Pharmacia panel of monoclonal antibody reagents (Launch diagnostics, Kent, UK) ${ }^{12}$ and the nutritional requirement for proline, arginine, hypoxanthine, and uracil was determined. ${ }^{13}$

\section{Genotypic analysis}

Template DNA was prepared as follows: a $1 \mu \mathrm{l}$ loopful of $N$ gonorrhoeae was suspended in $150 \mu \mathrm{l} 5 \%$ Chelex-100 resin slurry (Bio-Rad laboratories Ltd, Hemel Hempstead, UK) in distilled water. Samples were heated $\left(95^{\circ} \mathrm{C}\right.$ for 10 minutes $)$, centrifuged $\left(2\right.$ minutes at $13000 \mathrm{~g}$ ), and stored at $-20^{\circ} \mathrm{C}$ before use. Isolates were analysed retrospectively using NGMAST as described previously. ${ }^{9}$ Forward and reverse sequences of the por and $t b p B$ genes from each isolate were analysed, edited, and trimmed. A numeric sequence type (ST) was assigned based on the por and $t b p B$ alleles using the NGMAST website (www.ng-mast.net).

\section{Collection of epidemiological data and statistical analysis}

Epidemiological data, including the patients' sexual orientation, and the country where the gonococcal infection was acquired, were requested retrospectively for all isolates in this study. The statistical significance of associations between typing and epidemiological characteristics was tested using the $\chi^{2}$ test (psych.rice.edu/online_stat/analysis_lab/stat_ analysis.html).

\section{RESULTS}

Isolates from 818 episodes of gonococcal infection were submitted to the SNGRL during 2002, of which $11 \%$ (89) were fully resistant to ciprofloxacin and $2.1 \%$ (17) demonstrated reduced susceptibility to ciprofloxacin. A total of 106 isolates were analysed in this study.
Comparison of discrimination achieved by A/S typing and NG-MAST (fig 1)

The 106 isolates were divided into 17 auxotype/serovar (A/S) classes, seven of which were represented by single isolates, while the remaining ten A/S classes contained 2-38 isolates; the largest group of 38 isolates was the A/S class NR/Bropt. Three A/S classes contained isolates that exhibited reduced susceptibility to ciprofloxacin as well as isolates that exhibited resistance.

In contrast, the 106 isolates were divided by NG-MAST into 44 sequence types (STs), of which 32 were represented by single isolates and the remaining 12 STs contained between two and 32 isolates; the largest group of 32 isolates was ST147. No single ST was represented by a mixture of isolates with reduced susceptibility and resistance to ciprofloxacin; nine groups contained isolates that were fully resistant to ciprofloxacin, (STs 147, 203, 211, 278, 304, 307, 310, 311, and 314), and three groups contained isolates that had reduced susceptibility to ciprofloxacin (STs 66, 84, and 154). Ten of the 12 STs that included multiple isolates were homogeneous in A/S class. The remaining two STs were each represented by isolates that had the same serotype and that were auxotrophic (non-requiring), with the exception of one isolate, which had a single amino acid requirement (arginine or proline). Plasmid encoded resistance (as indicated by a positive nitrocephin test for penicillinase producing $N$ gonorrhoeae (PPNG) and a tetracycline MIC $\geqslant 8 \mathrm{mg} / \mathrm{l}$ for tetracycline resistant $N$ gonorrhoeae (TRNG)) was uniform for all isolates with the same ST, with the exception of STI47 where four isolates were PPNG and the remaining 28 had a negative nitrocephin test and therefore were not PPNG.

Isolates grouped together by $\mathrm{A} / \mathrm{S}$ class were all further subdivided by NG-MAST; the A/S class NR/Arst was the most heterogeneous, containing 16 isolates, which were subdivided into 11 different STs including eight that were unique. The majority (6/7) of isolates with a unique A/S class also had a unique ST, whereas 25/32 isolates with unique STs were grouped together with other isolates by A/S classification. Based on these 106 isolates, NG-MAST was more discriminatory that $\mathrm{A} / \mathrm{S}$ typing and resolved isolates into clusters that in most cases were homogeneous in their other characteristics.

\section{Distribution within Scotland of gonococcal isolates by ST (fig 2)}

Of the 106 isolates, $80(75.5 \%)$ were from patients in the three major metropolitan areas of Scotland: 42 from Greater Glasgow,

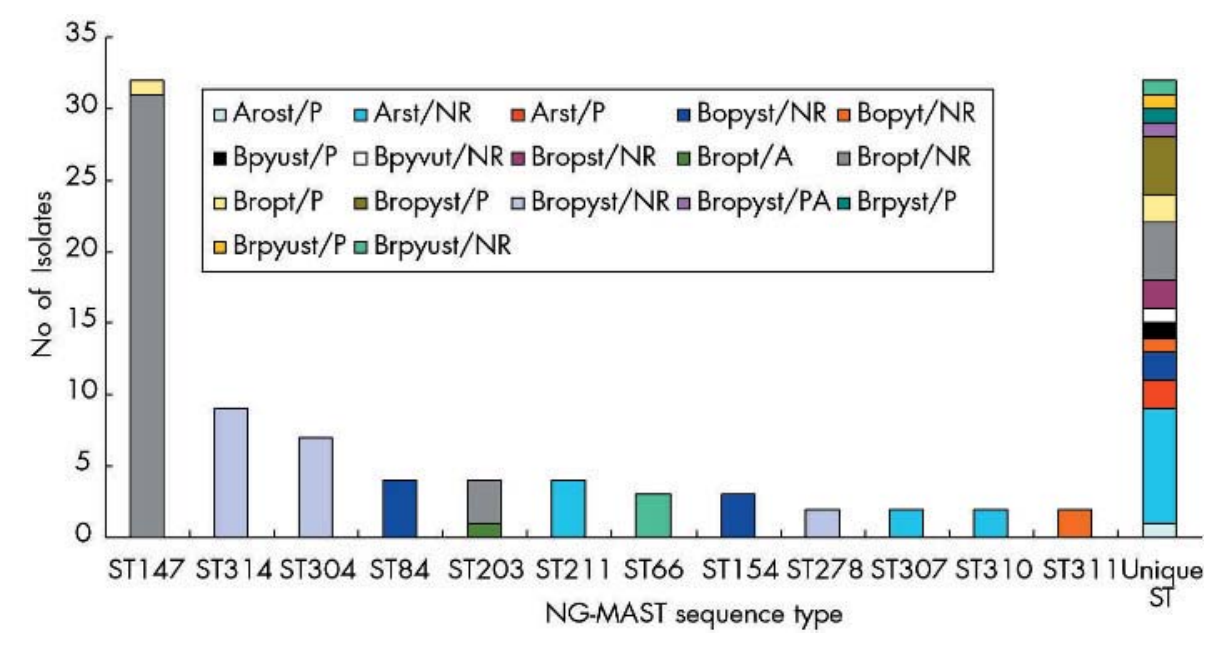

Figure 1 Comparison of discrimination achieved by $\mathrm{A} / \mathrm{S}$ typing and NG-MAST. 


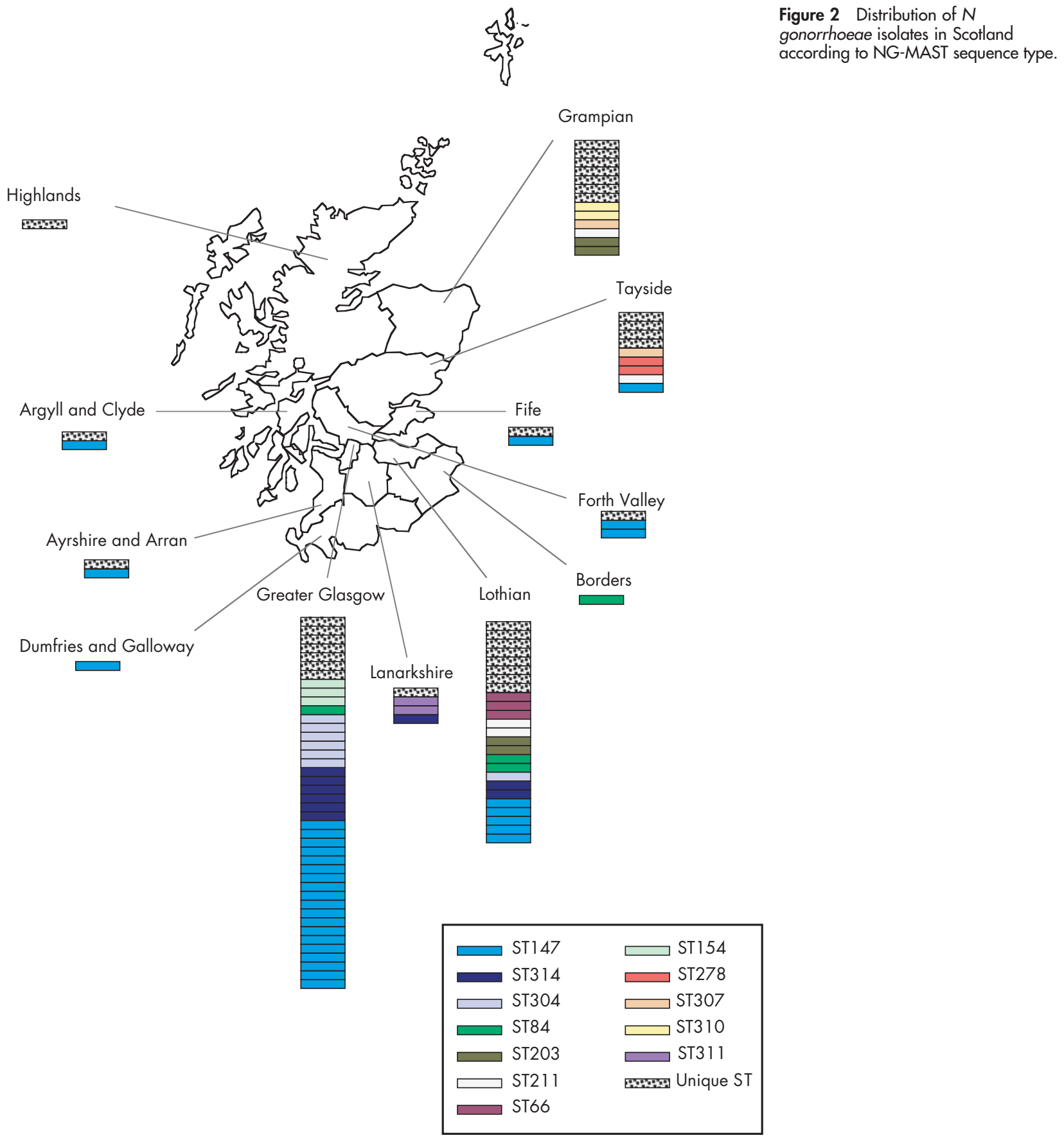

25 from Lothian (principally Edinburgh), and 13 from Grampian (principally Aberdeen). Isolates with the unique STs accounted for the minority of isolates in Greater Glasgow, 7/42 (16.7\%), and in Lothian, 8/25 (32\%), whereas in Grampian the majority of isolates had unique STs $(7 / 13,53.8 \%)$. The largest cluster of isolates was STI47, which occurred predominantly in Greater Glasgow and Lothian, but was present in six of the other 10 regions. Four of the six largest clusters occurred in the neighbouring areas of Greater Glasgow and Lothian.

\section{Association of ST and country of acquisition of gonorrhoea (table 1)}

The country of acquisition was known for 89 of 106 isolates, 55 of which $(51.9 \%)$ were acquired within the United
Kingdom. Clusters of isolates sharing the same ST were predominantly associated with transmission within the United Kingdom, whereas the majority of isolates with unique STs were associated with foreign travel $\left(\chi^{2}=28.2\right.$, $\mathrm{p}<0.0001)$. One notable exception was a cluster of four isolates (ST211), three of which were acquired in Thailand (this information was not available for the fourth). Most of the isolates originating abroad were acquired in the South East Asia and Pacific regions (28/34, 82.3\%). Six isolates were associated with travel within the United Kingdom to England; these were identified as STs 203 (no city specified), 147 (two isolates, from Liverpool and Manchester), 66 (from London), 84 (from Manchester), and 314 (from Scarborough). 
Table 1 Association of NG-MAST sequence type with the country of acquisition of gonorrhoea and sexual preference of the patient

\begin{tabular}{|c|c|c|c|c|c|c|c|}
\hline \multirow[b]{2}{*}{$\begin{array}{l}\text { NG-MAST } \\
\text { sequence type }\end{array}$} & \multicolumn{4}{|c|}{ Area of acquisition } & \multicolumn{3}{|c|}{ Patient sexual preference } \\
\hline & UK & $\begin{array}{l}\text { European, } \\
\text { non-UK }\end{array}$ & $\begin{array}{l}\text { SE Asia and } \\
\text { Pacific region* }\end{array}$ & Not given & MSW† & MSM $\ddagger$ & Not given \\
\hline ST147 & 24 & 1 & $1(1)$ & 6 & 22 & 8 & 2 \\
\hline ST314 & 6 & 1 & 0 & 2 & 8 & 0 & $\overline{1}$ \\
\hline ST304 & 6 & 0 & 0 & 1 & 7 & 0 & 0 \\
\hline ST84 & 2 & 0 & 1 & 1 & 0 & 3 & 1 \\
\hline ST203 & 3 & 0 & 0 & 1 & 4 & 0 & 0 \\
\hline ST211 & 0 & 0 & $3(3)$ & 1 & 3 & 1 & 0 \\
\hline ST66 & 3 & 0 & 0 & 0 & 0 & 3 & 0 \\
\hline ST154 & 2 & 1 & 0 & 0 & 1 & 2 & 0 \\
\hline ST278 & 0 & 0 & $1(1)$ & 1 & 0 & 0 & 2 \\
\hline ST307 & 1 & 0 & $1(1)$ & 0 & 2 & 0 & 0 \\
\hline ST310 & $1 \S$ & 0 & $1(1)$ & 0 & 2 & 0 & 0 \\
\hline ST311 & $1 \S$ & 0 & $1(1)$ & 0 & 2 & 0 & 0 \\
\hline Unique ST & $6 \S$ & 3 & $19(9)$ & 4 & 25 & 3 & 4 \\
\hline
\end{tabular}

\section{Association of ST cluster and patient sexual preference (table 1)}

The sexual preference of 96/106 (90.6\%) patients was known, the majority of whom were heterosexual. Three STs (147, 154, and 211) were represented by isolates from both heterosexual patients and MSM, whereas most other ST clusters were associated exclusively with either heterosexual or homosexual transmission.

\section{DISCUSSION}

This study demonstrates that NG-MAST achieves a greater degree of discrimination than A/S typing. Isolates within ST clusters were broadly homogeneous with respect to the phenotypic characteristics analysed, except for the ST147 cluster, which contained both PPNG and non-PPNG isolates. This observation is not unexpected, since plasmids are mobile genetic elements and may be lost or acquired independently of chromosomal genetic change. Indeed, the loss of resistance plasmids has previously been associated with fluoroquinolone use and developing gonococcal resistance. ${ }^{14}$

The epidemiological data demonstrate that in 2002 gonococcal infection from isolates with reduced susceptibility or resistance to ciprofloxacin occurred predominantly in the three largest metropolitan areas of Scotland (Glasgow, Edinburgh, and Aberdeen) and that these infections were acquired mainly by heterosexual transmission. Around half the infections were acquired within the United Kingdom and half were associated with foreign travel.

A more detailed description of the epidemiology is suggested by the NG-MAST data. NG-MAST identified eight clusters of between three and 32 isolates, indicating that multiple transmission networks exist. Isolates within NGMAST clusters occurred mainly in Glasgow and Edinburgh but not Aberdeen, and were predominantly associated with transmission within the United Kingdom, with six STs (66, $84,154,203,211$, and the largest, ST147) previously described in London. ${ }^{15}$ Two clusters of nine (ST314) and seven (ST304) isolates have not previously been observed. There were specific epidemiological links between six isolates from five ST clusters with English cities, demonstrating that these sexual networks extend beyond the main focus of Glasgow and Edinburgh. Most ST clusters were transmitted exclusively among homosexuals or heterosexuals, although within the largest cluster (ST147) both routes of transmission occurred, as was the case for isolates of this ST in London during 2000-2002. In London, transmission of ST147 isolates was predominantly by MSM, ${ }^{15}$ whereas in Scotland mainly heterosexual transmission occurred. Isolates with unique STs were predominantly associated with foreign travel and occurred in most areas of Scotland.

These data suggest that during 2002 there was sustained transmission of several UK acquired ciprofloxacin resistant gonococcal strains within networks in the two neighbouring population centres of Glasgow and Edinburgh, some of which were connected with networks occurring in London and other UK cities. By contrast, in Aberdeen there was no evidence of sustained transmission, with mainly single or paired isolates of any given ST occurring. Aberdeen is a major centre for the oil industry and this could account for a higher proportion of imported strains. The differences in transmission characteristics between different regions in Scotland highlighted in this study should aid specific interventions to be targeted as appropriate for the different geographical areas.

One ST cluster of four isolates appears unusual, in that three of four isolates were associated with travel to Thailand and transmission was via both homosexual (one case, acquired in Thailand) and heterosexual (three cases) routes. Further typing using another discriminatory typing system, such as opa typing, could be used to confirm the validity of this cluster. It is likely that these isolates are linked by sex tourism, with well known centres of commercial sex work being sought out independently by these travellers. In the absence of more detailed epidemiology, it is impossible to draw firm conclusions; however, the discriminatory power of NG-MAST and ease of data comparison between laboratories indicates its value for international strain comparison in the future.

This study demonstrates the power of combining epidemiological data with a highly discriminatory typing tool. NGMAST offers practical advantages, providing objective data defined by numerical descriptors and stored in a web based database. Already NG-MAST data have indicated links between clusters of isolates in London, Glasgow, and Edinburgh, which are substantiated by some of the patients' epidemiological details. The use of NG-MAST by both major centres of gonococcal surveillance in the United Kingdom (Sexually Transmitted Bacteria Reference Laboratory (STBRL) and SNGRL) will allow a more coherent approach to epidemiological investigation of gonorrhoea in the United Kingdom in the future. In addition, routine use of NG-MAST for all isolates received was recently introduced in the SNGRL 
with a rapid turnaround time and feedback of data to clinicians; this now offers the potential for responsive local epidemiology and appropriately targeted intervention as outbreaks of specific strains of gonorrhoea are identified.

\section{ACKNOWLEDGEMENTS}

Thanks are extended to all of our laboratory and clinical colleagues throughout Scotland for submitting isolates, for providing epidemiological information and for their enthusiasm and interest in this work. We thank David Aanensen for developing the NG-MAST website which is supported by the Wellcome Trust.

\section{CONTRIBUTORS}

HY initiated the study; IMCM and HMP carried out practical work; HMP performed data analysis; IMCM, CAI, and BGS were responsible for development of the NG-MAST method and establishment of the website for analysis; all authors contributed to the manuscript preparation.

\section{Authors' affiliations}

H M Palmer, H Young, Scottish Neisseria gonorrhoeae Reference Laboratory, Department of Medical Microbiology, Royal Infirmary of Edinburgh, 51 Little France Crescent, Edinburgh EH16 5SA, UK I M C Martin, C A Ison, Department of Infectious Diseases and Microbiology, Imperial College London, St Mary's Campus, London W2 IPG, UK

I M C Martin, B G Spratt, Department of Infectious Diseases Epidemiology, Imperial College London, St Mary's Campus, London W2 IPG, UK

I M C Martin, C A Ison, Sexually Transmitted Bacteria Reference Laboratory, Centre for Infections, Health Protection Agency, 61 Colindale Avenue, London NW9 5HT, UK

\section{REFERENCES}

1 Tapsall J. Antimicrobial resistance in $\mathrm{N}$ gonorrhoeae (www.emro.who.int/ asd/BackgroundDocuments/Neisseria_gonorrhoeae.pdf 2001).

2 Fenton KA, Ison C, Johnson AP, et al. Ciprofloxacin resistance in Neisseria gonorrhoeae in England and Wales in 2002. Lancet 2003;361:1867-9.

3 Young H, Palmer H, Winter A. Ciprofloxacin resistant gonorrhoea: the situation in Scotland and implications for therapy. SCIEH Wkly Rep 2003;37:178-80

4 Arreaza L, Salcedo C, Alcala B, et al. Antibiotic resistance of Neisseria gonorrhoeae in Spain: trends over the last two decades. J Antimicrob Chemother 2003;51:153-6.

5 Herida M, Sednaoui P, Goulet V. Gonorrhoea surveillance system in France: 1986-2000. Sex Transm Dis 2004;31:209-14.

6 Centers for Disease Control, Prevention (CDC). Increases in fluoroquinoloneresistant Neisseria gonorrhoeae among men who have sex with men-United States, 2003, and revised recommendations for gonorrhea treatment, 2004. Morb Mortal Wkly Rep 2004;53:335-8

7 Hobbs MM, Alcorn TM, Davis RH, et al. Molecular typing of Neisseria gonorrhoeae causing repeated infections: evolution of porin during passage within a community. J Infect Dis 1999;179:371-81.

8 Viscidi RP, Demma JC. Genetic diversity of Neisseria gonorrhoeae housekeeping genes. J Clin Microbiol 2003;41:197-204.

9 Martin IC, Ison C, Aanensen DM, et al. Rapid sequence-based identification of gonococcal transmission clusters in a large metropolitan area. J Infect Dis 2004; 189: 1497-505.

10 Young H, Patterson IC, McDonald DR. Rapid carbohydrate utilization test for the identification of Neisseria gonorrhoeae. Br J Vener Dis 1976;52:172-5.

11 Young $\mathbf{H}$, Moyes A, Roberston DHH, et al. Gonococcal infection within Scotland: antigenic heterogeneity and antibiotic susceptibility of infecting strains. Eur J Epid 1990;6:1-8.

12 Coghill DV, Young H. The serological classification of Neisseria gonorrhoeae with monoclonal antibody coagglutination reagents. Genitourin Med 1987;63:225-32.

13 Copley CG, Egglestone SI. Auxotyping of Neisseria gonorrhoeae isolated in the United Kingdom. J Med Micro 1983;16:295-302

$14 \mathrm{Kam} \mathrm{KM}$, Lo KK, Ng KY, et al. Rapid decline in penicillinase-producing Neisseria gonorrhoeae in Hong Kong associated with emerging 4fluroquinolone resistance. Genitourin Med 1995;71:141-4. 